\title{
High order FE and covariant description for contact problems
}

\author{
Alexander Konyukhov* and Karl Schweizerhof \\ Germany, D-76128, Karlsruhe, Kaiserstrasse 12, University of Karlsruhe, Institute of Mechanics
}

\begin{abstract}
The covariant contact description is applied to incorporate the treatment of contact problems into the high-order finite element technique. A hierarchical enrichment of the Ansatz space allows to construct a contact layer finite element combining both an exact geometry for contact surfaces, and a mesh with linear Ansatz functions for the interior of the contacting bodies. Good correlation with the Hertz problem is shown even within a few contact elements for the contact zone, however, not in general.
\end{abstract}

() 2006 WILEY-VCH Verlag GmbH \& Co. KGaA, Weinheim

\section{Introduction}

Within the modeling of contact problems using low order finite elements a problem concerning the discontinuity of contact tractions at neighboring element boundaries is arising. Various smoothing techniques to overcome this problem have been developed. These techniques are mainly based on covering surfaces discretized by finite elements with patches possessing proper smoothness (e.g. Bezier splines, NURB splines), see Wriggers [1], Laursen [2]. However, these approaches are not representing the exact geometry of contact bodies, e.g. there is a void between the smooth surface and the finite element surface. The influence of this assumption is not yet discussed in the literature. In order to investigate this two problems have to be solved. The first problem is that the geometry of the contact bodies must be represented exactly. The second problem is that the contact description must be independent of the order of surface approximations. One powerful method to satisfy the exact geometry is to apply high order finite elements, see Rank et. al. [3]. As an additional element a covariant contact description, developed in [4], [5] gives us a powerful tool to derive all necessary parameters for the iterative solution independently of the order of approximations of contact surfaces. Thus, the aim of the current presentation is to combine a high order finite element technique with a covariant description of contact problems.

\section{Structure of contact layer finite element}

We consider separately contact between a deformable body and a rigid base, leading to the situation contact layer - rigid surface finite element, and contact between two deformable bodies, leading to the situation contact layer - contact layer finite element. A hierarchical anisotropic enrichment of polynomial degree is proposed keeping the conformity with the linear FE mesh for the interior, see Fig. 1 for the 2D case. The anisotropic shape function space in this case consists of 8 vertex shape functions representing the nodal degrees of freedom; $p^{e_{l}}-1$ and $p^{e_{r}}-1$ edge shape functions of polynomial degree $p^{e_{l}}$ and $p^{e_{r}}$ for the left and right edges respectively; $\left(p^{b}-1\right) \times\left(p^{b}-1\right)$ bubble shape functions. The edge and side shape functions are constructed hierarchically based on integrated Legendre polynomials. Conformity with a linear mesh is preserved by keeping the linear approximation along the upper line and, if necessary, by setting the corresponding edge shape function to be linear. In addition the blending function method is exploited to represent the exact geometry of the contact line $A C B$, see [3] and references herein. The covariant approach is used to embed the contact in the surface element. Then, all contact parameters

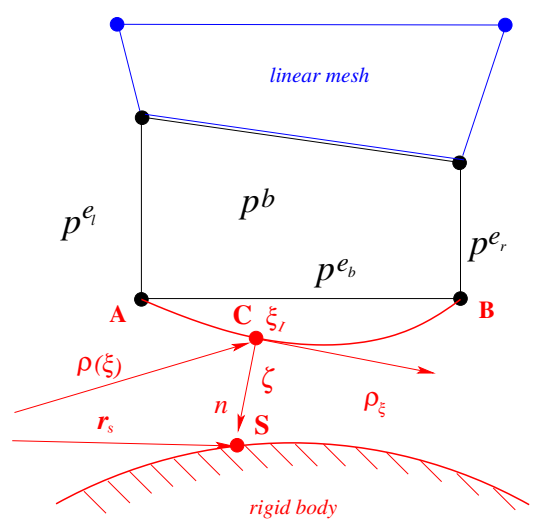

Fig. 1 Contact layer - rigid surface finite element. Geometry.

\footnotetext{
* Corresponding author: e-mail: Konyukhov@ifm.uni-karlsruhe.de, Phone: +49(0)721 608 3715, Fax: +49 (0)721 6087990
} 
are defined in the following curvilinear coordinate system placed on the contact surface:

$$
\mathbf{r}_{s}(\alpha)=\boldsymbol{\rho}(\xi)+\mathbf{n} \zeta
$$

where $\zeta$ is a value of penetration in normal direction $\mathbf{n}$. Vector $\mathbf{r}_{s}(\alpha)$ is representing a rigid surface for the contact layer - rigid surface finite element, and a surface of another enhanced finite element for the contact layer - contact layer finite element. For the rigid surface contact the value of penetration $\zeta$ and the remaining contact parameters are computed in Gauss points $\xi_{i}$ of the master surface. In case of the contact layer - contact layer element, all parameters are computed in the points of projected Gauss points taken on the slave surface $\mathbf{r}_{s}(\alpha)$. The results of the linearization can be directly taken from the covariant description in [4], [5]. In addition, only the approximation operator $\boldsymbol{\rho}(\xi)=\mathbf{A}(\mathbf{x})$ must be linearized, where $\mathbf{x}$ is a vector representing the exact geometry of the contact surfaces. The final finite element implementation follows closely the results given in [4] and [6].

\section{Numerical results}

The known analytical solution of the Hertz problem, the case of a cylinder on a plate, is chosen to test the proposed approach. Only one layer of finite elements 1-6 with linear shape function are modified into the contact layer - rigid surface finite elements, see Fig. 2.

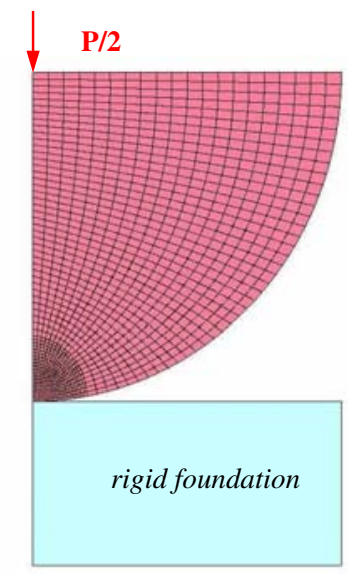

a)

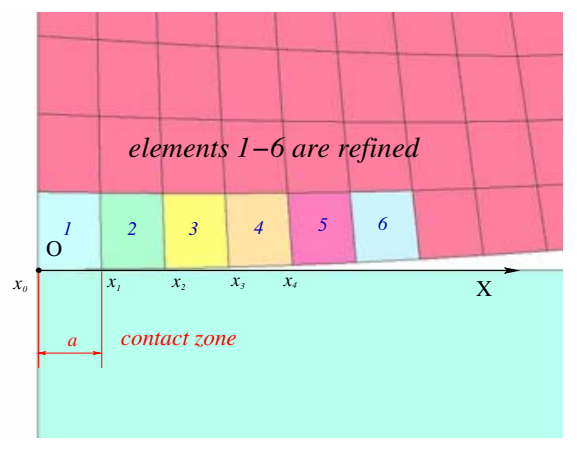

b)

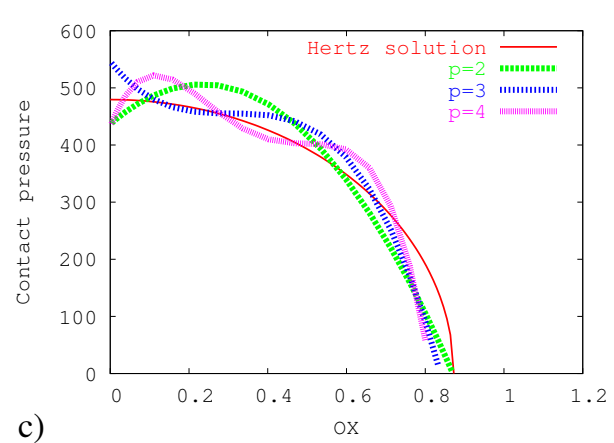

c)

Fig. 2 a) Hertz problem. b) Geometry and contact zone with enhanced contact layer finite elements. c) Distribution of contact pressure. Different polynomial order $p$ of contact layer element in the case of only single element in contact $x \approx a$.

As an interesting result, a fair correlation with contact pressure given by the Hertz solution is found even if the contact zone $a$ is within a single element, see Fig. 2. However, considerable oscillations may occur, when the contact zone is not closely aligned to the surface discretization even with a rather fine mesh.

\section{Conclusions}

In the current contribution an algorithmic embedding of contact into a high order finite element scheme with exact representation of the geometry has been developed. The approach is based on the covariant description for contact problems allowing to take into account any approximation of the contact surfaces. Particular attention is paid to a contact layer finite element combining the linear finite mesh for the interior and anisotropically refined elements only for one so-called contact layer.

Acknowledgements We thank the DFG for the support given by grant SCHW 307/18-2.

\section{References}

[1] P. Wriggers, Computational Contact Mechanics. (Wiley, Chichester, 2002).

[2] T. A. Laursen, Computational Contact and Impact Mechanics. (Springer, New York, 2002).

[3] E. Rank, A. Düster, V. Nübel, K. Preusch, O.T. Bruhns, High order finite elements for shells. Comput. Meth. Appl. Mech. Eng. 194, 2494-2512 (2005).

[4] A. Konyukhov, K. Schweizerhof, Contact formulation via a velocity description allowing efficiency improvements in frictionless contact analysis. Comput. Mech. 33, 165-173, (2004).

[5] A. Konyukhov, K. Schweizerhof, Covariant description for frictional contact problems. Comput. Mech. 35, 190-213, (2005).

[6] M. Harnau, A. Konyukhov, K. Schweizerhof, Algorithmic aspects in large deformation contact analysis using "Solid-Shell" elements. Comput. Struct. 83, 1804-1823, (2005). 